Case Report

\title{
Angiomyolipoma of the Thoracic Wall: An Extremely Rare Diagnostic Challenge
}

\author{
Georgios Gemenetzis, ${ }^{1}$ Eleni Kostidou, ${ }^{1}$ Kalliroi Goula, ${ }^{2}$ \\ Vassilios Smyrniotis, ${ }^{1}$ and Nikolaos Arkadopoulos ${ }^{1}$ \\ ${ }^{1}$ 4th Department of Surgery, Medical School of Athens, Attikon University Hospital, Rimini 1, Attiki, 12462 Athens, Greece \\ ${ }^{2}$ Department of Pathology, Medical School of Athens, Attikon University Hospital, Rimini 1, Attiki, 12462 Athens, Greece \\ Correspondence should be addressed to Georgios Gemenetzis; georgegemen@gmail.com
}

Received 15 June 2014; Accepted 13 August 2014; Published 8 September 2014

Academic Editor: Marcello Picchio

Copyright (C) 2014 Georgios Gemenetzis et al. This is an open access article distributed under the Creative Commons Attribution License, which permits unrestricted use, distribution, and reproduction in any medium, provided the original work is properly cited.

\begin{abstract}
Extrarenal angiomyolipoma (AML) is an extremely uncommon lesion, accounting for less than $9 \%$ of all angiomyolipomas. We present a previously unreported case of a rarely located gigantic extrarenal angiomyolipoma at the posterolateral chest wall of a 35-year-old woman. Clinically, the lesion had all the characteristics of a benign tumor, being soft in palpation, painless, and growing in size in a slow rate. Histologically, the lesion consisted of convoluted thick-walled blood vessels without an elastic layer, interlacing fascicles of smooth muscle, and mature adipose tissue, features consistent with an angiomyolipoma. The mass was surgically removed, without any postoperative complications, and the patient has an uneventful postoperative course. Signs of local recurrence have not been observed. The purpose of this brief report is to point out the necessity of including angiomyolipoma in the differential diagnosis of adipose layer lesions.
\end{abstract}

\section{Introduction}

Angiomyolipoma (AML) is a clonal mesenchymal tumour with malignant potential, usually located at the kidney and most commonly found in middle-aged women. Renal angiomyolipoma is strongly associated with tuberous sclerosis complex (TSC), a group of autosomal dominant genetic disorders, and lymphangioleiomyomatosis. It is an invasive and recurring lesion, is prone to spontaneous bleeding, and consists of variable portions of interlacing fascicles of epithelioid smooth muscle, convoluted thick-walled blood vessels, and mature fat. Extrarenal manifestation of angiomyolipoma is uncommon and has been mostly reported in the liver. It is very rare and presents with male predominance, no association with TSC or lymphangioleiomyomatosis, and no signs of local recurrence. Extrarenal angiomyolipoma is also solitary and well circumscribed and is characterized by the lack of epithelioid tissue. The purpose of this report is to describe a case of angiomyolipoma in the posterior chest wall with a diameter of $22 \mathrm{~cm}$, a location and size that have never been reported previously in the literature.

\section{Case Presentation}

The patient is a 35-year-old, otherwise healthy, white female, who presented to our clinic with a large tender mass in the left posterolateral chest wall. The physical examination revealed a solitary and painful nodule adherent to deep layers and covered with normal skin. The patient's medical history included a resection of a cystic formation of the right lung in childhood. She did not receive any systemic treatment and had no family history of tuberous sclerosis complex or other inherited conditions. MRI was performed and the existence of a $22 \mathrm{~cm}$ lobular mass was confirmed. It was located just over the lower limit of the left scapula and extended posterolaterally roughly down to the rim of the left 10th-11th rib (Figure 1). The lesion's attributes on the MRI images advocated for a hemangioma-like lesion. The mass was surgically removed, without any postoperative complications. The specimen's histopathologic report described a mass measuring $22 \times 15 \mathrm{~cm}$ with solid and cystic characteristics. Microscopically, a histological image of angiomyolipoma was observed. The diagnosis was based on the composition of the 
TABLE 1: Locations of extrarenal manifestations of angiomyolipomas reported in the literature.

\begin{tabular}{|c|c|c|c|c|}
\hline Location & Study & Age & Gender & Max diameter $(\mathrm{cm})$ \\
\hline \multirow{5}{*}{ Ear and periauricular area } & Argenyi et al. [4] & 67 & $\mathrm{M}$ & 1 \\
\hline & Val-Bernal and Mira [5] & 49 & M & 2.5 \\
\hline & Lee et al. [6] & 32 & M & 1.5 \\
\hline & Büyükbabani et al. [7] & 38 & M & 2.5 \\
\hline & Shin et al. [8] & 26 & $\mathrm{~F}$ & 1 \\
\hline \multirow{3}{*}{ Extremities } & Rodriguez-Fernandez and Caro-Mancilla [9] & 58 & M & 4 \\
\hline & DeBloom et al. [10] & 50 & $\mathrm{~F}$ & 3 \\
\hline & Makino et al. [11] & 16 & $\mathrm{~F}$ & 2.5 \\
\hline Nose & Büyükbabani et al. [7] & 36 & $\mathrm{M}$ & 1.5 \\
\hline \multirow{4}{*}{ Adrenals } & Godara et al. [12] & 45 & $\mathrm{~F}$ & 15 \\
\hline & Kong et al. [13] & 61 & M & 10 \\
\hline & Yener and Ozcelik [14] & 45 & $\mathrm{~F}$ & 6 \\
\hline & $\mathrm{Hu}$ and $\mathrm{Xi}[15]$ & 55 & $\mathrm{~F}$ & 16 \\
\hline \multirow{3}{*}{ Torso } & Morita et al. [16] & 56 & $\mathrm{~F}$ & 2 \\
\hline & Ammanagi et al. [17] & 3 & $\mathrm{~F}$ & 2.5 \\
\hline & Gemenetzis et al. (2014) & 35 & $\mathrm{~F}$ & 22 \\
\hline
\end{tabular}

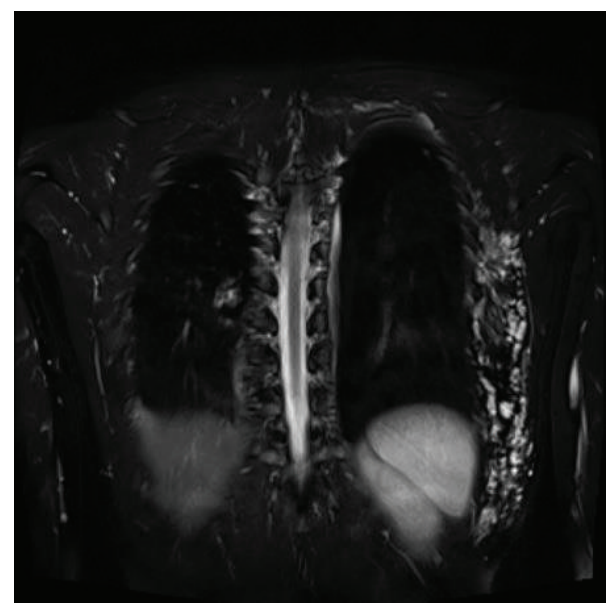

FIGURE 1: MRI scan (coronal view) of the lesion, which is located under the left scapula and extending posterolaterally down to the rim of the left 10th rib.

tumour, which consisted of convoluted thick-walled blood vessels without an elastic layer, interlacing fascicles of smooth muscle, and mature adipose tissue (Figure 2). Features of malignancy were not observed. The lesion tested negative for reaction to $\mathrm{HMB}-45$ antibody, an immunohistochemical test to rule out epithelioid tumour involvement. Twelve months after surgery the patient is free of disease and the lesion has not presented any signs of local recurrence.

\section{Discussion}

Originally, angiomyolipoma was known as a hamartomatous lesion. It is now considered to be a mesenchymal tumour with malignant potential [1], usually observed in the kidney and very often (25-50\%) accompanying the tuberous sclerosis complex (TSC) [2]. TSC is a group of autosomal

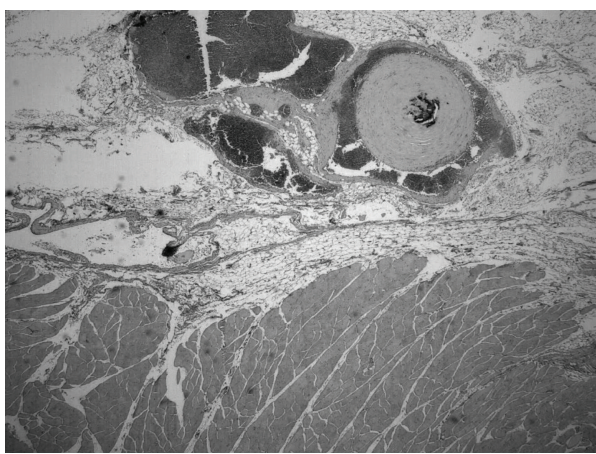

Figure 2: H-E staining (10x) that highlights the three distinct components of the angiomyolipoma: thick-walled blood vessels, mature adipose tissue, and interlacing smooth muscle fibers.

dominant genetic disorders caused by germline mutations in the TSC1 and TSC2 genes, located on chromosomes 9q and $16 \mathrm{p}$, respectively, and characterized by hamartomatous lesions in multiple organs [3]. The extrarenal manifestation of angiomyolipoma, apart from liver, is extremely rare (Table 1) and not associated with TSC, as is the case with our patient. Extrarenal angiomyolipomas usually present with clinical features similar to those of a lipoma, a hemangioma-like lesion, or an epidermal cyst, which are all included in the differential diagnosis.

MRI imaging is the most important diagnostic tool in distinguishing angiomyolipomas, since the proportion of fat in the lesion defines the ability for imaging. Macroscopic fat has high signal intensity on $\mathrm{T} 1$ and $\mathrm{T} 2$ sequence and leads to high sensitivity in the detection of extrarenal angiomyolipomas [18]. According to Brimo et al. [19] angiomyolipomas can be classified, depending on the consistence, in three major categories: regular, epithelioid with atypia, and epithelioid without atypia. Renal angiomyolipomas belong to the second 
or third group. The vast majority of reported extrarenal angiomyolipomas belong to the first group (except for one case reported by D'Antonio et al. [20]) and have as components mature adipose tissue, thick-walled blood vessels, and bundles of smooth muscle cells (each component forms more than $10 \%$ of the tumour). Most importantly, these lesions lack epithelioid components. Immunohistochemical features can also assist in distinguishing extrarenal angiomyolipomas. Particularly, HMB-45 antibody is positive in renal angiomyolipomas and none of the extrarenal tumors are reactive. Our lesion was also HMB-45 negative, thus consistent with these reports.

The angiomyolipoma diagnosed in our patient in the posterolateral chest wall is generally observed in middleaged men, is noninvasive, and shows no signs of recurrence or metastasis. The size of a regular angiomyolipoma defines the symptoms, since lesions with diameter less than four $\mathrm{cm}$ are usually asymptomatic, whereas those with diameter bigger than four $\mathrm{cm}$ may cause pain or lead to bleeding. The appropriate treatment for an extrarenal angiomyolipoma is surgical resection, whereas the absence of malignancy makes any adjuvant therapy unnecessary. The posterolateral chest wall has great extent and permits the presence of a sizeable angiomyolipoma (diameter of $22 \mathrm{~cm}$ ). Apparently, the location of the lesion plays a significant role in its size. A year after the surgical resection, our patient is healthy and has not presented any signs of local recurrence.

We presented a previously unreported case of a rarely located gigantic extrarenal angiomyolipoma of the posterolateral chest wall and pointed out that angiomyolipoma should be included in the differential diagnosis of adipose layer lesions.

\section{Consent}

Patient's written consent is available and can be provided if requested.

\section{Conflict of Interests}

The authors declare that there is no conflict of interests regarding the publication of this paper.

\section{References}

[1] L. Yang, X. L. Feng, S. Shen et al., "Clinicopathological analysis of 156 patients with angiomyolipoma originating from different organs," Oncology Letters, vol. 3, no. 3, pp. 586-590, 2012.

[2] O. Rouvière, H. Nivet, N. Grenier et al., "Kidney damage due to tuberous sclerosis complex: management recommendations," Diagnostic and Interventional Imaging, vol. 94, no. 3, pp. 225237, 2013.

[3] S. K. Rakowski, E. B. Winterkorn, E. Paul et al., "Renal manifestations of tuberous sclerosis complex: incidence, prognosis, and predictive factors," Kidney International, vol. 70, no. 10, pp. 1777-1782, 2006.

[4] Z. B. Argenyi, W. W. Piette, and J. A. Goeken, "Cutaneous angiomyolipoma: a light-microscopic, immunohistochemical, and electron-microscopic study," The American Journal of Dermatopathology, vol. 13, no. 5, pp. 497-502, 1991.

[5] J. F. val-Bernal and C. Mira, "Cutaneous angiomyolipoma," Journal of Cutaneous Pathology, vol. 23, no. 4, pp. 364-368, 1996.

[6] P. Y. Lee, C. C. Wu, M. C. Wen et al., "Hepatic angiomyolipoma: a report of two cases," Zhonghua Yi Xue Za Zhi, vol. 57, no. 1, pp. 74-78, 1996.

[7] N. Büyükbabani, S. Tetikkurt, and A. S. Öztürk, "Cutaneous angiomyolipoma: report of two cases with emphasis on HMB45 utility," Journal of the European Academy of Dermatology and Venereology, vol. 11, no. 2, pp. 151-154, 1998.

[8] J.-U. Shin, K.-Y. Lee, and M. R. Roh, "A case of a cutaneous angiomyolipoma," Annals of Dermatology, vol. 21, no. 2, pp. 217220, 2009.

[9] A. Rodriguez-Fernandez and A. Caro-Mancilla, "Cutaneous angiomyolipoma with pleomorphic changes," Journal of the American Academy of Dermatology, vol. 29, no. 1, pp. 115-116, 1993.

[10] J. R. DeBloom, A. Friedrichs, B. L. Swick, and D. C. Whitaker, "Management of cutaneous angiomyolipoma and its association with tuberous sclerosis," Journal of Dermatology, vol. 33, no. 11, pp. 783-786, 2006.

[11] E. Makino, J. Yamada, J. Tada, J. Arata, and K. Iwatsuki, "Cutaneous angiolipoleiomyoma," Journal of the American Academy of Dermatology, vol. 54, no. 1, pp. 167-171, 2006.

[12] R. Godara, M. G. Vashist, S. L. Singla et al., "Adrenal angiomyolipoma: a rare entity," Indian Journal of Urology, vol. 23, no. 3, pp. 319-320, 2007.

[13] C. H. Kong, I. Mohamed Rose, P. Singam, G. E. Hong, L. B. Cheok, and Z. M. Zainuddin, "Angiomyolipoma of the adrenal gland: a case report," Iranian Red Crescent Medical Journal, vol. 12, no. 4, pp. 489-491, 2010.

[14] O. Yener and A. Ozcelik, "Angiomyolipoma of the right adrenal gland," ISRN Surgery, vol. 2011, Article ID 102743, 2 pages, 2011.

[15] H. Hu and X. Xi, "Giant adrenal angiomyolipoma," Journal of Clinical Endocrinology and Metabolism, vol. 97, no. 11, pp. 38353836, 2012.

[16] K. Morita, Y. Shida, K. Shinozaki et al., "Angiomyolipomas of the mediastinum and the lung," Journal of Thoracic Imaging, vol. 27, no. 1, pp. W21-W23, 2012.

[17] A. S. Ammanagi, V. D. Dombale, and V. V. Shindholimath, "Cutaneous angiomyolipoma," Indian Dermatology Online Journal, vol. 3, no. 1, pp. 40-41, 2012.

[18] G. Low, K. Sahi, and H. Dhliwayo, "Low T2 signal intensity on magnetic resonance imaging: a feature of minimal fat angiomyolipomas," International Journal of Urology, vol. 19, no. 1, pp. 90-91, 2012.

[19] F. Brimo, B. Robinson, C. Guo, M. Zhou, M. Latour, and J. I. Epstein, "Renal epithelioid angiomyolipoma with atypia: a series of 40 cases with emphasis on clinicopathologic prognostic indicators of malignancy," The American Journal of Surgical Pathology, vol. 34, no. 5, pp. 715-722, 2010.

[20] A. D'Antonio, A. Caleo, and O. Caleo, "Monotypic epithelioid angiomyolipoma of the adrenal gland: an unusual site for a rare extrarenal tumor," Annals of Diagnostic Pathology, vol. 13, no. 5, pp. 347-350, 2009. 


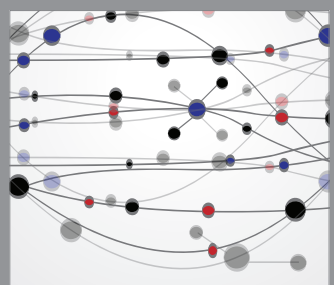

The Scientific World Journal
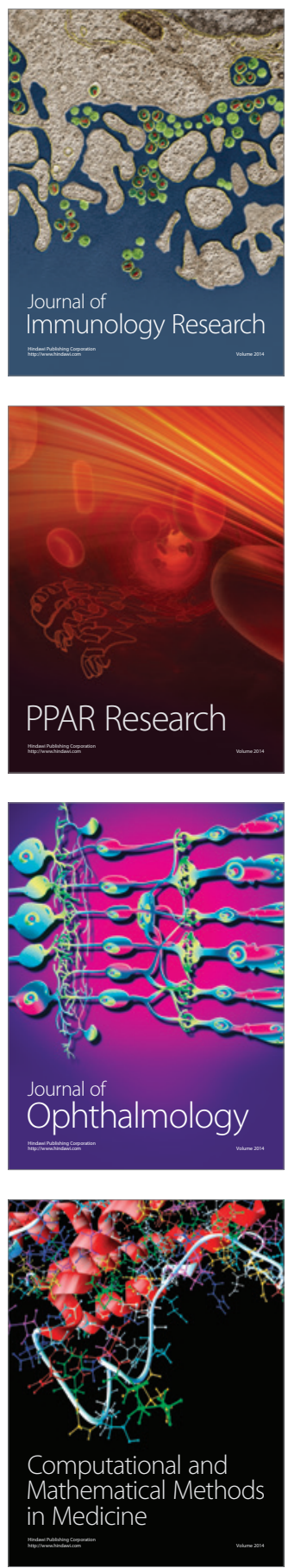

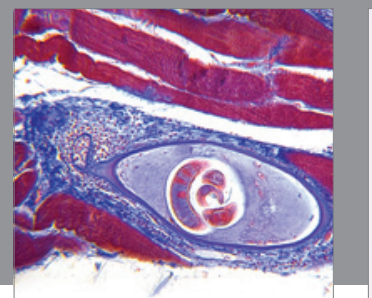

Gastroenterology

Research and Practice
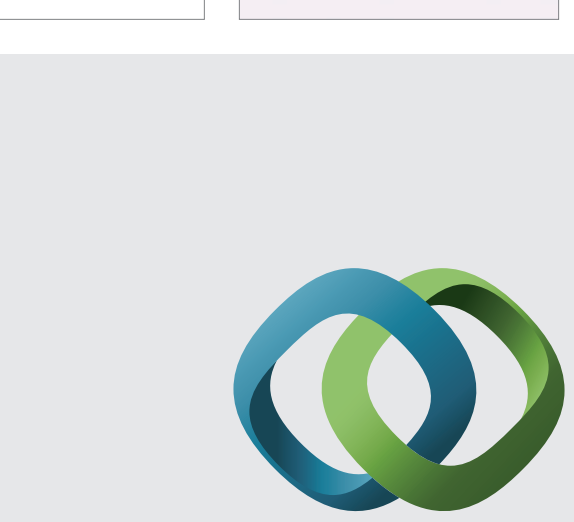

\section{Hindawi}

Submit your manuscripts at

http://www.hindawi.com
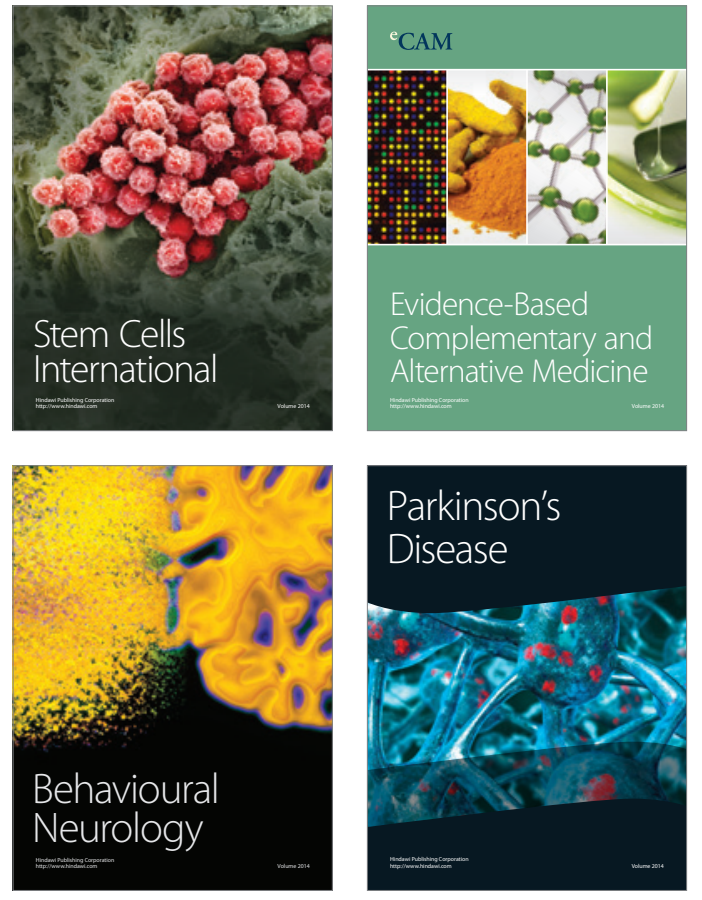
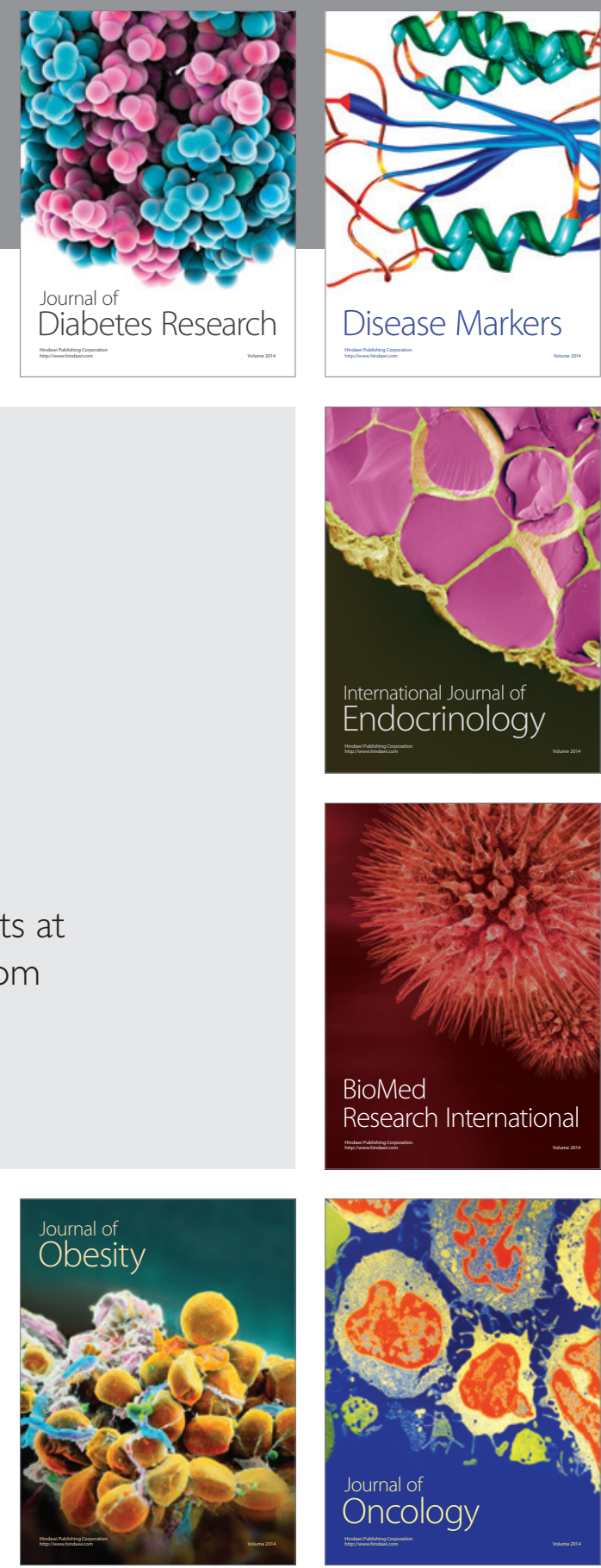

Disease Markers
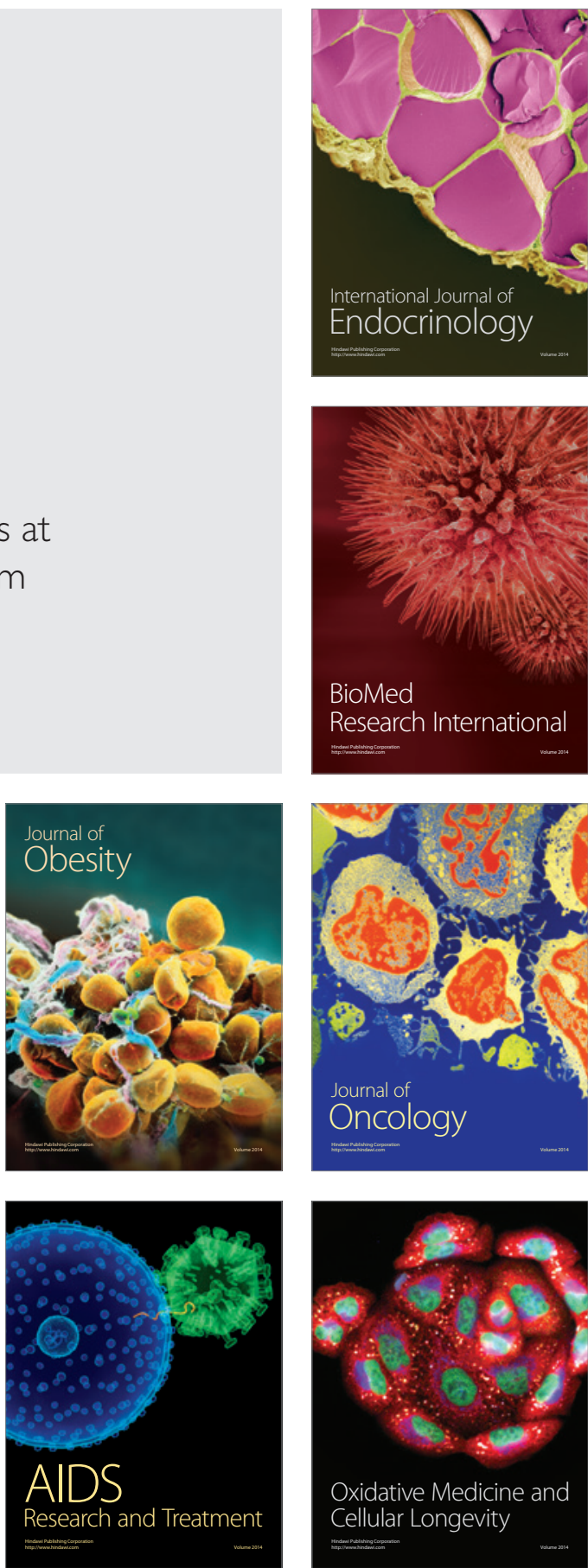